\title{
Clinical significance of velamentous cord insertion prenatally diagnosed in twin pregnancy
}

Hyun-mi Lee ${ }^{1}$, SiWon Lee ${ }^{2}$, Min-Kyung Park ${ }^{3}$, You Jung Han ${ }^{4}$, Moon Young Kim ${ }^{4}$, Hye Yeon Boo ${ }^{1}$, Jin Hoon Chung $^{5 *}$

${ }^{1}$ Department of Obstetrics and Gynecology, CHA Ilsan Medical Center, CHA University, Goyang, Korea

${ }^{2}$ Department of Obstetrics and Gynecology, Mount Sinai Medical Center, Miami Beach, FL, USA

${ }^{3}$ Department of Obstetrics and Gynecology, Yonsei University College of Medicine, Seoul, Korea

${ }^{4}$ Department of Obstetrics and Gynecology, CHA Gangnam Medical Center, CHA University, Seoul, Korea

${ }^{5}$ Department of Obstetrics and Gynecology, University of Ulsan College of Medicine, Asan Medical Center, Seoul, Korea

Corresponding author:

Jin Hoon Chung, M.D., Ph.D.

Division of Maternal Fetal Medicine, Department of Obstetrics and Gynecology, University of Ulsan College of Medicine, Asan Medical Center, 88, Olympic-ro 43-gil, Songpa-gu, Seoul, Republic of Korea

Tel: 82-2-3010-3654

Email: sabi0515@hanmail.net

\section{Abstract \\ Background}

The purpose of this study was to evaluate the prevalence of velamentous cord insertion (VCI) and the actual association between pathologically confirmed VCI and perinatal outcomes in twins based on the chorionicity.

\section{Methods}

All twin pregnancies who received prenatal care at a specialty clinic for multiple pregnancies, from less than 12 weeks of gestation until delivery in a single institution between 2015 and 2018 were included in this retrospective cohort study.

\section{Results}

A total of the 941 twins were included in the study. The prevalence of VCI in dichorionic (DC) twins and monochorionic diamniotic (MCDA) twins was $5.8 \%$ and $7.8 \%$, respectively $(p=0.251)$. In all study population, the prevalence of vasa previa and placenta accreta were higher in VCI group compared to that of non-VCI group ( $p=0.008$ and 0.022). In MCDA twins with VCI, birth weight, 1 and 5-minute Apgar score were lower compared to DC twins with VCI $(p=0.010,0.002$ and 0.000$)$. There was no significant association between VCI and selective fetal growth restriction $(p=0.708)$, twin-to-twin transfusion syndrome $(p=0.400)$ and birth-weight discordance $(>20 \%$ and $>25 \%)(p=0.378$ and 0.161$)$ in MCDA twins.

\section{Conclusion}

VCI in twins was not a risk factor for adverse perinatal outcomes and twin-specific complications.

\section{Keywords}

Velamentous cord insertion; Twin; Perinatal outcomes; Pregnancy outcomes; Chorionicity; Twin specific complications 


\section{Introduction}

Velamentous cord insertion (VCI), defined as an abnormal insertion of the umbilical vessels that insert into the fetal membranes before entering the placenta is one of the abnormal findings of placental insertion of the umbilical cord.

The incidence of VCI has been reported to be $0.1-1.8 \%$ in all pregnancies [1]. An increased prevalence of VCI had been reported for twin pregnancies (1.6-40\%) especially in monochorionic (MC) twin pregnancies, probably as a result of unequal sharing of the placentas [2, 3].

A number of previous studies have reported that VCI is associated with adverse maternal and perinatal outcomes [1, 4, 5]. Likewise, VCI in MC twins has been proposed as a risk factor in the development of selective fetal growth restriction (sFGR), twin-to-twin transfusion syndrome (TTTS) and birth-weight (BW) discordance [4, 6-9]. However, some studies were incongruent with the results and suggested that the adverse complications in VCI were possibly due to the selection bias and pointed out that these unique adverse outcomes in $\mathrm{MC}$ twins are likely the consequences of the vascular complications of the placental sharing rather than VCI $[4,10,11]$.

Therefore, the aim of this study was to assess the prevalence of VCI in twin pregnancy and evaluate whether prenatally detected VCI was associated with adverse pregnancy outcomes including unique complications of twin pregnancies based on the chorionicity.

\section{Material and methods}

This was a retrospective cohort study of twin pregnancies with known pregnancy outcomes, who were diagnosed with VCI during prenatal ultrasound in Cheil General Hospital and Women's Healthcare Center between January 2015 and March 2018. The chorionicity was determined by the first trimester ultrasonography by number of gestational sacs, yolk sacs and fetuses. The monochorionic monoamniotic (MCMA) twin pregnancies with high risk of perinatal complications and twin pregnancies with major congenital anomaly or aneuploidy were excluded. Ethical approval of the study was obtained from the institutional review board (CGH-IRB-2018-37). The need to obtain informed consent was waived.

Placenta cord insertion site was categorized into two groups: VCI and non-VCI. VCI was determined during the anatomy ultrasound by a maternal fetal medicine (MFM) specialist using high-resolution sonography equipments (Voluson E8 [GE Healthcare, Milwaukee, WI], EPIQ5 [Philips, Netherlands])). As part of this examination, a targeted scan of the placental umbilical cord insertion site using the appropriate magnification and settings was also performed. Once the umbilical cord was identified, it was followed until it reached the placental surface, the insertion site being further confirmed with color flow imaging if necessary. The diagnosis of VCI was made when the umbilical cord was clearly attached to the placental membranes instead of the placental body. When VCI was detected, the patient underwent sonography again 2 weeks later by another MFM specialist. The scan was performed either transabdominally or transvaginally (Figure 1) [12]. VCI group was defined as VCI in one or both of the fetuses. The chorionicity was determined by assessing the number of the gestational sac, yolk sac, and the shape of intertwin membranes on the first trimester ultrasound [13]. The VCI and the chorionicity were confirmed by postpartum pathological examination of the placenta (Figure 2).

Clinical significance of VCI in twin pregnancies were evaluated between VCI group versus non-VCI group and based on the chorionicity. Pregnancy and neonatal outcomes including, preeclampsia, gestational diabetes mellitus (GDM), preterm premature rupture of membranes (PPROM), placenta previa, vasa previa, placenta accreta, postpartum hemorrhage, birth weight (BW) discordance of more than $20 \%$ and $25 \%$, birth weight (BW) of the neonates, twin-to-twin transfusion syndrome (TTTS), selective fetal growth restriction (sFGR), 1 and 5minute Apgar score and neonatal intensive care unit (NICU) admission were analyzed between the groups.

BW discordance was calculated by subtracting the weight of the smaller twin from the weight of larger twin and then dividing by the weight of larger twin, expressed as a percentage. sFGR was diagnosed when the birthweight of one of the twins was below the $10^{\text {th }}$ centile for gestational age and there was at least $25 \%$ difference in estimated body weight between the fetuses in MC twin [14]. Diagnosis of TTTS was made according to the established criteria, $\mathrm{MC}$ twin and discordant amniotic fluid volume (AFV) (MVP $>8 \mathrm{~cm}$ in the recipient twin, MVP $<2 \mathrm{~cm}$ in the donor twin) [15].

Statistical analyses were performed using the software SPSS for windows version 17.0 (SPSS,Inc.,Chicago,Illinois,USA). The Chi-square test was used to assess the association between the fetal level data aggregated and pair level data with VCI. All significance tests were two-tailed. A $P$-value of less than 0.05 was considered statistically significant for all analyses. 


\section{Results}

A total of 981 twin pregnancies were identified during the study period. After excluding 40 pregnancies with major congenital anomaly $(n=7)$, chromosomal abnormalities $(n=5)$, MCMA twins $(n=5)$ or lost to follow-up $(n=23), 941$ twins (DC, $n=788$ and MCDA, $n=153)$ were enrolled.

Total 58 cases $(6.2 \%)$ of VCIs were diagnosed by ultrasound, including 46 cases (5.8\%) of DC twin pregnancies and 12 cases $(7.8 \%)$ of MCDA twin pregnancies. There was no statistically significant difference in the prevalence of VCI between DC and MCDA twin pregnancies $(p=0.251)$ in our study.

Maternal characteristics and perinatal outcomes of the twin pregnancies in VCI group compared to non-VCI groups are presented in Table 1 . The two groups did not differ regarding maternal age at delivery $(p=0.128)$, pregnancies conceived via assisted reproductive technique (ART) $(p=0.969)$, gestational age at delivery $(p=0.077)$, preeclampsia $(p=0.395), \operatorname{GDM}(p=0.643)$, PPROM $(p=0.466)$ and BW discordance $(>20 \%$ and $>25 \%)$ $(p=0.759$ and 0.621$)$. VCI-group showed significantly higher rates of vasa previa $(p=0.008)$ and placenta accreta ( $p=0.022)$ compared to non-VCI group. There was no difference in neonatal outcomes including birthweight, 1 and 5-minute Apgar score and the rate of NICU admission between VCI and non-VCI groups.

Table 2 shows the maternal characteristics, pregnancy and neonatal outcomes in VCI group based on the chorionicity. There were no differences in maternal characteristics or pregnancy outcomes regarding maternal age at delivery $(p=0.325)$, gestational age at delivery $(p=0.078)$ and pregnancy outcomes including preeclampsia $(p=0.968), \operatorname{GDM}(p=0.290), \operatorname{PPROM}(p=0.953)$, placenta previa $(p=0.364)$, vasa previa $(p=0.825)$, placenta accreta $(p=0.364)$, BW discordance $(>20 \%$ and $>25 \%)(p=0.549$ and 0.061$)$ and the rate of NICU admission $(p=0.111)$ based on the chorionicity. However, lower BW $(p=0.010)$, and lower 1and 5-minute Apgar score $(p=0.002$ and 0.000$)$ were noted in MCDA twin pregnancy group compared to DC twin pregnancy group, despite no difference in gestational age at delivery.

When DC twin pregnancies were subdivided into VCI and non-VCI groups (Table 3), the two groups did not differ regarding maternal age at delivery $(p=0.106)$, gestational age at delivery $(p=0.196)$, preeclampsia $(p=0.714), \operatorname{GDM}(p=0.876), \operatorname{PPROM}(p=0.693)$, BW discordance $(>20 \%$ and $>25 \%)(p=0.582$ and 0.897$)$ and

neonatal outcomes including birth weight, 1 and 5-minute Apgar score and the rate of NICU admission. Notably, vasa previa and placenta accrete were more common in VCI group than non-VCI group in DC twin pregnancies $(p=0.018$ and $p=0.018)$.

Table 4 presents the comparison of pregnancy outcomes in VCI and non-VCI group in MCDA twin pregnancies. VCI group did not differ from non-VCI group regarding maternal age at delivery $(p=0.576)$, gestational age at delivery $(p=0.323)$ and pregnancy outcomes including preeclampsia $(p=0.254)$, GDM $(p=0.378), \operatorname{PROM}(p=0.378)$, placenta previa $(p=0.240)$, vasa previa $(p=0.240), \mathrm{BW}(p=0.816), 1$ and 5-minute Apgar score $(p=0.123$ and 0.283$)$, and the rate of NICU admission $(p=0.938)$ in MCDA twin pregnancies. Furthermore, there was no significant association between VCI and sFGR $(p=0.708)$, TTTS $(p=0.400)$ and BW discordance $(>20 \%$ and $>25 \%)(p=0.378$ and 0.161$)$ in MCDA twin pregnancies.

\section{Discussion}

We evaluated the prevalence and associated adverse pregnancy outcomes of VCI in twin pregnancies based on the chorionicity. The incidence of VCI in total twin pregnancies was $6.2 \%$ and the incidence of VCI in DC and MCDA twins were $5.8 \%$ and $7.8 \%$, respectively. The incidence of VCI in our study was lower compared to the previous studies $[1,4,16]$. Also, no significant difference in prevalence was noted between DC and MCDA twin pregnancies $(p=0.251)$ in our study.

Another notable finding of this study was that when VCI and non-VCI groups were compared based on the chorionicity, VCI group did not show significant association with adverse pregnancy outcomes. Furthermore, VCI was not associated with twin specific complications including sFGR, TTTS and BW discordance in MCDA twin pregnancies.

Many previous studies described the association between VCI and adverse pregnancy/ perinatal outcomes [1, 4, 6-8]. VCI was associated with increased risk of fetal growth restriction, preterm labor, low Apgar scores, placental abruption, fetal and neonatal death [5, 16-18]. In MCDA twin pregnancy, when unequal sharing of the placental territory is present, the higher association between VCI and adverse outcomes such as sFGR, TTTS and BW discordance had been described [1, 3, 6-8, 19, 20]. However, several studies with relatively large sample sizes came to a contradictory conclusion about the association between abnormal cord insertion in MCDA twin pregnancies and BW discordance/sFGR [21, 22] and several studies also demonstrated no significant impact of VCI on TTTS $[6,21,23]$. Similarly, we did not observe significant differences in neonatal outcomes between VCI group and non-VCI group including BW, Apgar scores at 1 and 5-minute, and NICU admission rates in twin pregnancies. Our results are consistent with the previous studies [21, 22], which 
demonstrated no association between VCI and the risk of sFGR and BW discordance in MCDA twins. Furthermore, in our study, VCI group in MCDA twin pregnancy did not show association with TTTS, even if we included 2 cases with VCI that were transferred to a tertiary center for fetoscopic laser surgery. In particular, we included twin pregnancies with VCI only, unlike previous study which included marginal cord insertion as well as VCI and found association between abnormal cord insertion and sFGR, TTTS and BW discordance in MCDA twins [23].

In MCDA twin pregnancies with VCI, birth weight, which may be a major factor of neonatal mortality, and Apgar scores at 1 and 5-minute were lower than DC twins with VCI. This may be due to the natural characteristic of MC twin pregnancy, which is unequal sharing of the placental territory, not VCI, considering that there was no difference in the gestational age at delivery between MC and DC twin pregnancies with VCI.

In this study, twin pregnancies with VCI demonstrated higher incidence of vasa previa and placenta accrete than those with non-VCI. Although, previous studies report higher risk of vasa previa in multiple pregnancies with VCI $[1,4,24]$, our study showed no difference in the prevalence of vasa previa when analyzed based on the chorionicity.

Strengths of the study include that only twin pregnancies who underwent consistent prenatal care with MFM specializing in multiple pregnancies and delivered in the same institution were enrolled. Secondly, as this specialty clinic for multiple pregnancies include all multiple pregnancies with or without complications, unlike most of the tertiary care center with referred complicated pregnancies, this study may reflect general twin pregnancy population. The main limitation of the study was that this was a retrospective study, and TTTS of stage II or higher had to be sent to a tertiary center, because fetoscopic laser coagulation was not performed in this institution, so the outcomes related to this were excluded from the analysis. However, as mentioned above, there was no association between VCI and TTTS even when the cases with TTTS of stage II or higher were included. Rather, it was considered that selection bias would be small because it was targeted to twin pregnancies who had been prenatal care at the institution since the early pregnancy.

In conclusion, our study demonstrated that VCI in twin pregnancies did not have a significant association with adverse pregnancy outcomes, although vasa previa and placenta accrete were observed at a higher rate in twin pregnancies with VCI. The results of this study may be helpful when counseling twin pregnancies diagnosed with VCI on prenatal ultrasound and avoid unnecessary medical interventions or concerns. In additions, meticulous follow-up will be required for the presence or absence of vasa previa to determine timing and mode of delivery.

Funding

No funding was obtained

Authors' contributions

JHC designed this study. YJH, MYK and HYB contributed to the acquisition of data. MKP contributed to analysis of data. SWL and JHC reviewed articles. HML wrote the initial manuscript. All authors contributed to the writing of the final manuscript.

\section{Details of ethics approval}

The study was approved by the institutional review board (CGH-IRB-2018-37).

Conflict of interest

None of the authors have any conflicts of interest to disclose.

\section{References}

1. Raisanen, S.; Georgiadis, L.; Harju, M.; Keski-Nisula, L.; Heinonen, S. Risk factors and adverse pregnancy outcomes among births affected by velamentous umbilical cord insertion: a retrospective population-based register study. Eur J Obstet Gynecol Reprod Biol. 2012, 165, 231-234; DOI: 10.1016/j.ejogrb.2012.08.021.

2. Buchanan-Hughes, A; Bobrowska, A; Visintin, C; Attilakos, G; Marshall, J. Velamentous cord insertion: results from a rapid review of incidence, risk factors, adverse outcomes and screening. Syst Rev. 2020, 9, 147; DOI: $10.1186 / \mathrm{s} 13643-020-01355-0$.

3. Cambiaso, O; Zhao, D.P.; Abasolo, J.I.; Aiello, H.A.; Oepkes, D.; Lopriore, E.; Otano, L. Discordance of cord 
insertions as a predictor of discordant fetal growth in monochorionic twins. Placenta. 2016, 47, 81-85; DOI: 10.1016/j.placenta.2016.09.009.

4. Ebbing, C.; Kiserud, T.; Johnsen, S.L.; Albrechtsen, S.; Rasmussen, S. Prevalence, risk factors and outcomes of velamentous and marginal cord insertions: a population-based study of 634,741 pregnancies. PLoS One. 2013, 8, e70380; DOI: 10.1371/journal.pone.0070380.

5. Eddleman, K.A.; Lockwood, C.J.; Berkowitz, G.S.; Lapinski, R.H.; Berkowitz, R.L. Clinical significance and sonographic diagnosis of velamentous umbilical cord insertion. Am J Perinatol. 1992, 9, 123-126; DOI: $10.1055 / \mathrm{s}-2007-994684$.

6. Kent, E.M.; Breathnach, F.M.; Gillan, J.E.; McAuliffe, F.M.; Geary, M.P.; Daly S.; Higgins, J.R.; Dornan, J.; Morrison, J.J.; Burke, G.; Higgins, S.; Carroll, S.; Dicker, P.; Manning, F.; Malone, F.D. Placental cord insertion and birth weight discordance in twin pregnancies: results of the national prospective ESPRiT Study. Am J Obstet Gynecol. 2011, 205, 376.e1-7; DOI: 10.1016/j.ajog.2011.06.077.

7. Hanley, M.L.; Ananth, C.V.; Shen-Schwarz, S.; Smulian, J.C.; Lai, Y.L.; Vintzileos, A.M. Placental cord insertion and birth weight discordancy in twin gestations. Obstet Gynecol. 2002, 99, 477-482; DOI: 10.1016/s0029-7844(01)01734-3.

8. Lopriore, E.; Sueters, M.; Middeldorp, J.M.; Oepkes, D.; Walther, F.J.; Vandenbussche, F.P. Velamentous cord insertion and unequal placental territories in monochorionic twins with and without twin-to-twin-transfusion syndrome. Am J Obstet Gynecol. 2007, 196, 159.e1-5; DOI: 10.1016/j.ajog.2006.10.865.

9. Yonetani, N.; Ishii, K.; Kawamura, H.; Mabuchi, A.; Hayashi, S.; Mitsuda, N. Significance of Velamentous cord insertion for twin-twin transfusion syndrome. Fetal Diagn Ther. 2015, 38, 276-281; DOI: $10.1159 / 000381639$.

10. Ebbing, C.; Kiserud, T.; Johnsen, S.L.; Albrechtsen, S.; Rasmussen, S. Third stage of labor risks in velamentous and marginal cord insertion: a population-based study. Acta Obstet Gynecol Scand. 2015, 94, 878883; DOI: 10.1111/aogs.12666.

11. Ebbing, C.; Johnsen, S.L.; Albrechtsen, S.; Sunde, I.D.; Vekseth, C.; Rasmussen, S. Velamentous or marginal cord insertion and the risk of spontaneous preterm birth, prelabor rupture of the membranes, and anomalous cord length: a population-based study. Acta Obstet Gynecol Scand. 2017, 96, 76-85; DOI: 10.1111/aogs.13035.

12. Sepulveda, W. Velamentous insertion of the umbilical cord: a first-trimester sonographic screening study. $J$ ultrasound Med. 2006, 25, 963-968; DOI: 10.7863/jum.2006.25.8.963.

13. Taylor, M.J.O. The management of multiple pregnancy. Early Hum Dev. 2006, 82, 365-370; DOI: 10.1016/j.earlhumdev.2006.03.008.

14. Gratacos, E.; Lewis, L.; Munoz, B.; Acosta-Rojas, R.; Hernandez-Andrade, E.; Martinez J.M.; Carreras, E.; Deprest, J. A classification system for selective intrauterine growth restriction in monochorionic pregnancies according to umbilical artery Doppler flow in the smaller twin. Ultrasound Obstet Gynecol. 2007, 30, 28-34; DOI: $10.1002 /$ uog.4046.

15. Quintero, R.A.; Morales, W.J.; Allen, M.H.; Bornick, P.W.; Johnson, P.K.; Kruger, M. Staging of twin-twin transfusion syndrome. J Perinatol. 1999, 19, 550-555; DOI: 10.1038/sj.jp.7200292.

16. Yerlikaya, G.; Pils, S.; Springer, S.; Chalubinski, K.; Ott, J. Velamentous cord insertion as a risk factor for obstetric outcome: a retrospective casecontrol study. Arch Gynecol Obstet. 2016, 293, 975-981; DOI: 10.1007/s00404-015-3912-x.

17. Heinonen, S.; Ryynanen, M.; Kirkinen, P.; Saarikoski, S. Perinatal diagnostic evaluation of velamentous umbilical cord insertion: clinical, Doppler, and ultrasonic findings. Obstet Gynecol. 1996, 87, 112-117; DOI: 10.1016/0029-7844(95)00339-8.

18. Ananth, C.V.; Savitz, D.A.; Williams, M.A. Placental abruption and its association with hypertension and prolonged rupture of membranes: a methodologic review and meta-analysis. Obstet Gynecol. 1996, 88, 309-318; DOI: 10.1016/0029-7844(96)00088-9.

19. De Paepe, M.E.; Shapiro, S.; Young, L.; Luks, F.I. Placental characteristics of selective birth weight 
discordance in diamniotic-monochorionic twin gestations. Placenta. 2010, 31, 380-386; DOI: 10.1016/j.placenta.2010.02.018.

20. Hack, K.E.A.; Nikkels, P.G.J.; Koopman-Esseboom, C.; Derks, J.B.; Elias, S.G.; van Gemert, M.J.C.; Visser, G.H.A. Placental characteristics of monochorionic diamniotic twin pregnancies in relation to perinatal outcome. Placenta. 2008, 29, 976-981; DOI: 10.1016/j.placenta.2008.08.019.

21. Naoto Yonetani; Keisuke Ishii; Hiroshi Kawamura; Aki Mabuchi; Shusaku Hayashi; Nobuaki Mitsuda. Significance of Velamentous Cord Insertion for Twin-Twin Transfusion Syndrome. Fetal Diagn Ther. 2015, 38, 276-281; DOI: 10.1159/000381639.

22. Costa-Castro, T.; Zhao, D.P.; Lipa, M.; Haak, M.C.; Oepkes, D.; Severo, M.; Montenegro, N.; Matias, A.; Lopriore, E. Velamentous cord insertion in dichorionic and monochorionic twin pregnancies - Does it make a difference? Placenta. 2016, 42, 87-92; DOI: 10.1016/j.placenta.2016.04.007.

23.Kalafat, E.; Thilaganathan, A.; Papageorghiou, A.; Bhide, A.; Khalil, A. Significance of placental cord insertion site in twin pregnancy. Ultrasound Obstet Gynecol. 2018, 52, 378-384; DOI: 10.1002/uog.18914.

24. Sullivan, E.A.; Javid, N.; Duncombe, G.; Li, Z.; Safi, N.; Cincotta, R.; Homer, C.S.E.; Halliday, L.; Oyelese, Y. Vasa previa diagnosis, clinical practice, and outcomes in Australia. Obstet Gynecol. 2017, 130, 591-598; DOI: 10.1097/AOG.0000000000002198. 